MATHEMATICS OF COMPUTATION

Volume 69 , Number 230 , Pages $861-866$

S 0025-5718(99)01105-9

Article electronically published on March 10, 1999

\title{
ZEROES OF DIRICHLET $L$-FUNCTIONS AND IRREGULARITIES IN THE DISTRIBUTION OF PRIMES
}

\author{
CARTER BAYS AND RICHARD H. HUDSON
}

\begin{abstract}
Seven widely spaced regions of integers with $\pi_{4,3}(x)<\pi_{4,1}(x)$ have been discovered using conventional prime sieves. Assuming the generalized Riemann hypothesis, we modify a result of Davenport in a way suggested by the recent work of Rubinstein and Sarnak to prove a theorem which makes it possible to compute the entire distribution of $\pi_{4,3}(x)-\pi_{4,1}(x)$ including the sign change (axis crossing) regions, in time linear in $x$, using zeroes of $L(s, \chi), \chi$ the nonprincipal character modulo 4 , generously provided to us by Robert Rumely. The accuracy with which the zeroes duplicate the distribution (Figure 1) is very satisfying. The program discovers all known axis crossing regions and finds probable regions up to $10^{1000}$. Our result is applicable to a wide variety of problems in comparative prime number theory. For example, our theorem makes it possible in a few minutes of computer time to compute and plot a characteristic sample of the difference $\operatorname{li}(x)-\pi(x)$ with fine resolution out to and beyond the region in the vicinity of $6.658 \times 10^{370}$ discovered by te Riele. This region will be analyzed elsewhere in conjunction with a proof that there is an earlier sign change in the vicinity of $1.39822 \times 10^{316}$.
\end{abstract}

\section{INTRODUCTION}

A rather vague comment made by P. L. Chebyshev in 1853 regarding an excess of primes of the form $4 n+3$ over those of the form $4 n+1$ gave rise to comparative prime number theory. In 1957, Leech [10] discovered the first two regions of integers with $\pi_{4,3}(x)<\pi_{4,1}(x)$. Subsequent regions were discovered by D. Lehmer and Bays and Hudson, see [1], 2]. A seventh region begins at $x=1,488,478,427,089$, and was found by Bays and Hudson in 1996.

In this paper we modify a result of Davenport, making it possible for us to compute, using the generalized Riemann hypothesis, the irregularities in the distribution of primes in all arithmetic progressions and the famous sign changes of $\pi(x)-\operatorname{li}(x)$, where

$$
\operatorname{li}(x)=\lim _{\epsilon \rightarrow 0}\left\{\int_{0}^{1-\epsilon} \frac{d t}{\log t}+\int_{1+\epsilon}^{x} \frac{d t}{\log t}\right\},
$$

using the zeroes of the real non-principal Dirichlet $L$-functions modulo $q$ (and the zeroes of $\zeta(s)$ ) in linear time (more precisely, the time is $\mathcal{O}(n x)$, where $n$ is the number of zeroes and $x$ is the length of the interval being searched). Our theorem is clearly useful for a wide variety of problems in comparative prime number theory,

Received by the editor March 17, 1997 and, in revised form, April 1, 1998 and July 6, 1998. 1991 Mathematics Subject Classification. Primary 11A15, 11M26, 11Y11, 11 Y35. 
including theoretical computations of logarithmic densities for Chebyshev's bias for all moduli for which zeroes have been computed, as well as the sign changes of $\operatorname{li}(x)-\pi(x)$.

In this note we show that all known regions with $\pi_{4,3}(x)<\pi_{4,1}(x)$ and, in fact, the entire distribution function $\pi_{4,3}(x)-\pi_{4,1}(x)$ can be duplicated with great accuracy from the zeroes of $L(s, \chi), \chi$ the nonprincipal character modulo 4 . Figure 1 clearly depicts this fact, giving all axis crossing regions which must occur if the generalized Riemann hypothesis holds and finding that they do, in fact, coincide with all known regions. The first undiscovered region, near 9 trillion, could be verified by direct computation in a few weeks on a fast computer. The computation, using our theorem, requires a few seconds. We have computed theoretical crossings for $x$ up to $10^{1000}$. In Figure 2 we display values where $\pi_{4,3}(x)-\pi_{4,1}(x)$ is near a minimum locally, using Theorem 1 , and compare them to known values; see [2].

We are deeply indebted to Robert Rumely for providing us with the zeroes of the $L$-functions. We have high confidence in the accuracy of his data, even though he has pointed out to us that that his computational method for producing zeroes has not been rigorously proven. Andrew Odlyzko also provided us with 101,052 zeroes of $\zeta(s)$.

The major contribution of this note is to provide the means for anyone with a reasonable knowledge of programming to study the irregularities in the distribution of primes in arithmetic progressions and the sign change regions of $\pi(x)-\operatorname{li}(x)$, obtaining results (under the GRH) which otherwise would not be obtainable. For example, using our algorithm, it takes thirty minutes to search for sign changes of $\pi(x)-\operatorname{li}(x)$ for $x<10^{400}$. The program rediscovers the sign change region in the vicinity of $6.658 \times 10^{370}$ found by te Riele [12]. These results will be refined and presented elsewhere, and a proof given that an earlier sign change does, in fact, occur in the vicinity of $1.39822 \times 10^{316}$ (see [3]).

\section{The AnAlytic THEORY}

Let $b=2^{\alpha_{0}} P_{1}^{\alpha_{1}} \cdots P_{k}^{\alpha_{k}}$ and let $\gamma(b)=2^{k+\beta-1}$, where $\beta=1$ if $\alpha_{0}=0$ or $1, \beta=2$ if $\alpha_{0}=2$, and $\beta=3$ if $\alpha_{0} \geq 3$. Then $\gamma(b)$ denotes the ratio of quadratic non-residues of $b$ to quadratic residues [11, p. 167]. Let $\Sigma_{N}(x, b)$ and $\Sigma_{R}(x, b)$ denote respectively the number of primes $\leq x$ in all progressions $b n+c$ with $c$ a quadratic non-residue of $b$ and in all progressions $b n+c^{\prime}$ with $c^{\prime}$ a quadratic residue of $b$. Although our results generalize to composite moduli not having a primitive root (with $\Sigma_{N}(x, b)-\Sigma_{R}(x, b)$ replaced by $\Sigma_{N}(x, b)-\gamma(b) \Sigma_{R}(x, b)$ ), we assume for simplicity here that $b=4, q^{\alpha}$, or $2 q^{\alpha}$, where $q$ is an odd prime. Assuming the generalized Riemann hypothesis, we prove the following:

Theorem 1. For $b=4, q^{\alpha}$, or $2 q^{\alpha}, x \geq 2, T \geq 1$, we have under the generalized Riemann hypothesis,

$$
\begin{aligned}
& \Sigma_{N}(x, b)-\Sigma_{R}(x, b) \\
& \quad=\pi\left(x^{\frac{1}{2}}\right) / 2+\pi\left(x^{\frac{1}{2}}\right) \sum_{0 \leq \gamma \leq T} \frac{\sin \gamma \log x}{\gamma}+\mathcal{O}_{x, T}\left(\frac{x(\log x+\log T)^{2}}{T(\log x)}+\frac{x^{\frac{1}{2}}}{\log ^{2} x}\right),
\end{aligned}
$$

where $\gamma$ runs over the imaginary parts of the non-trivial zeroes of $L(s, \chi)$, where $\chi$ is the real nonprincipal character. 
Proof. The proof is a straightforward adaptation of Davenport [5, p. 101] as presented by Rubinstein and Sarnak [13, p. 181] with minor modifications. In particular, (2.4) and (2.12) of [13] are essential.

Following Hudson [7], we normalize the difference on the left-hand-side of (2.1) using $2 / \pi\left(x^{\frac{1}{2}}\right)$ rather than $(\log x) / x^{\frac{1}{2}}$. Bays and Hudson have observed in [3], [6], and [7, p. 563] that terms after the first in

$$
\begin{aligned}
\frac{\operatorname{li} x^{\frac{1}{2}}}{2}= & \frac{x^{\frac{1}{2}}}{\log x}+\frac{2 x^{\frac{1}{2}}}{\log ^{2} x}+\frac{8 x^{\frac{1}{2}}}{\log ^{3} x}+\frac{48 x^{\frac{1}{2}}}{\log ^{4} x} \\
& +\cdots+\frac{2^{k_{2}-1}\left(k_{2}-1\right) ! x^{\frac{1}{2}}}{\log ^{k_{2}} x}+\mathcal{O}\left(\frac{x^{\frac{1}{2}}}{\log ^{k_{2}+1} x}\right),
\end{aligned}
$$

cannot be ignored in numerical problems in comparative prime number theory requiring precision.

Let $\chi$ be the real nonprincipal Dirichlet character and define

$$
H(x)=\frac{-2}{\pi\left(x^{\frac{1}{2}}\right)} \sum_{p \leq x} \chi(p)=\frac{2}{\pi\left(x^{\frac{1}{2}}\right)}\left(\Sigma_{N}(x, b)-\Sigma_{R}(x, b)\right),
$$

and in the aptly named " $b=1$ " case [13, p. 175], we take

$$
H(x)=\frac{2}{\pi\left(x^{\frac{1}{2}}\right)}(\operatorname{li}(x)-\pi(x)) .
$$

Assume the generalized Riemann hypothesis is true, and let $\gamma$ run over the imaginary parts of the non-trivial zeroes of $L(s, \chi)$ if $b=4, q^{\alpha}$, or $2 q^{\alpha}$, and over the non-trivial zeroes of $\zeta(s)$ in the " $b=1$ " case (in the upper half-plane).

Appealing to Rubinstein and Sarnak ((2.12) on page 181 of [13]), we have

$$
H(x)=1+\sum_{\gamma \leq T} \frac{x^{i \gamma}}{\frac{1}{2}+i \gamma}+\mathcal{O}_{q}\left(\frac{x^{\frac{1}{2}} \log ^{2} x T}{T}+\frac{1}{\log x}\right) .
$$

Setting $x=e^{y}$ and appealing to Davenport [5, p. 101] (see also (2.4) of [13]), we have

$$
\sum_{\gamma} \frac{1}{\left(\frac{1}{4}+\gamma^{2}\right)}<\infty
$$

so that $\left(\right.$ since $\left.\log ^{2} x T=(\log x+\log T)^{2}=(y+\log T)^{2}\right)$

$$
H\left(e^{y}\right)=1+2 \sum_{0 \leq \gamma \leq T} \frac{\sin \gamma y}{\gamma}+\mathcal{O}\left(\frac{e^{\frac{y}{2}}(y+\log T)^{2}}{T}+\frac{1}{y}\right)
$$

or, equivalently,

$$
H(x)=1+2 \sum_{0 \leq \gamma \leq T} \frac{\sin \gamma \log x}{\gamma}+\mathcal{O}\left(\frac{x^{\frac{1}{2}}(\log x+\log T)^{2}}{T}+\frac{1}{\log x}\right) .
$$

Finally, multiplying by $\left(\pi\left(x^{\frac{1}{2}}\right)\right) / 2$, we have at once $(2.1)$, completing the proof of the theorem. 


\section{THE COMBINATORIAL THEORY}

Unfortunately, the analytic theory alone does not readily give us $\mathcal{O}$ constants which are useful in numerical work. We also do not have the luxury of fixing $x$ and letting $T \rightarrow \infty$ so that the first part of the $\mathcal{O}$-term in (2.1) vanishes. Fortunately, we have a combinatorial theory, Hudson and Bays [6, pp. 85-91], to which to appeal. To the extent that there are no biases present apart from the Chebyshev bias, so that oscillations of

$$
\Sigma_{N}(x, b)-\Sigma_{R}(x, b) \quad \text { about } \pi\left(x^{\frac{1}{2}}\right) / 2
$$

can be expected to be equidistributed (the oscillations above $\pi\left(x^{\frac{1}{2}}\right) / 2$ effectively cancelling the oscillations below [6] p. 88]), the error in (2.1) arises from the $x^{\frac{1}{2}} / \log ^{2} x$ part of the $\mathcal{O}$-term. Indeed, as pointed out to the authors by Rubinstein, the oscillating term summed over all nonprincipal characters, which denotes the demarcation from the mean [13, p. 179], is $\mathcal{O}\left(x^{\frac{1}{2}} / \log ^{2} x\right)$, though the constant is not known. If, however, the oscillations of $\Sigma_{N}(x, b)-\Sigma_{R}(x, b)$ are evenly distributed about $\pi\left(x^{\frac{1}{2}}\right) / 2$, and we use the approximation $\pi\left(x^{\frac{1}{2}}\right) \sim$ li $x^{\frac{1}{2}}$, we have from (5.11) of [6] that

$$
\begin{aligned}
\pi\left(x^{\frac{1}{2}}\right) \sim \operatorname{li} x^{\frac{1}{2}}=\frac{2 x^{\frac{1}{2}}}{\log x} & +\frac{4 x^{\frac{1}{2}}}{\log ^{2} x}+\frac{16 x^{\frac{1}{2}}}{\log ^{3} x}+\frac{96 x^{\frac{1}{2}}}{(\log x)^{4}} \\
& +\cdots+\frac{2^{k}(k-1) ! x^{\frac{1}{2}}}{(\log x)^{k}}+\mathcal{O}\left(\frac{\left(x^{\frac{1}{2}}\right)}{(\log x)^{k+1}}\right)
\end{aligned}
$$

and the implied constant $c$ in (2.4) associated with the $1 / \log x$ term should be close to 1 , assuming that the $\pi\left(x^{\frac{1}{2}}\right) / 2$ term arising in both the combinatorial and analytic theory is the dominant term. Terms after the first on the right-hand-side of (3.1), and lower order terms arising from Riemann's formula and the analogous formula for the modulus 4 (see, e.g. [9]), since they are at most $4 x^{\frac{1}{2}} / \log ^{2} x$, contribute so little that the values found in Figure 2 applying Theorem 1 with $c=1$ and 12,000 zeroes of $L(s, \chi)$ agree closely with values computed using the sieve discussed in [4]. Using this as our working hypothesis, we computed Figures 1 and 2.

Remark. We are deeply indebted to the referee for pointing out several deficiencies in the original manuscript. Since the manuscript was first submitted, we have strengthened the numerical evidence supporting the choice of $c=1$ for the constant associated with the $1 / \log x$ part of the $\mathcal{O}$ term in $(2.4)$ in several ways. The computed value for $\pi_{4,1}(x)-\pi_{4,3}(x)$ at $x=18,699,356,321$ differs from the theoretical model by only 24 ( -2719 versus -2743$)$, and even this difference is reduced if terms after the second in (3.1) are taken into account. Even more significantly, we have computed the Chebyshev bias for the modulus 4 using the above model and, at $10^{218}$, find that (after including a small correction factor recently found by Rubinstein and Hudson) the convergence of the bias to .9959 ..., see [13, p. 188], agrees to four decimal places. The result and similar bias findings will be presented in a forthcoming paper. 

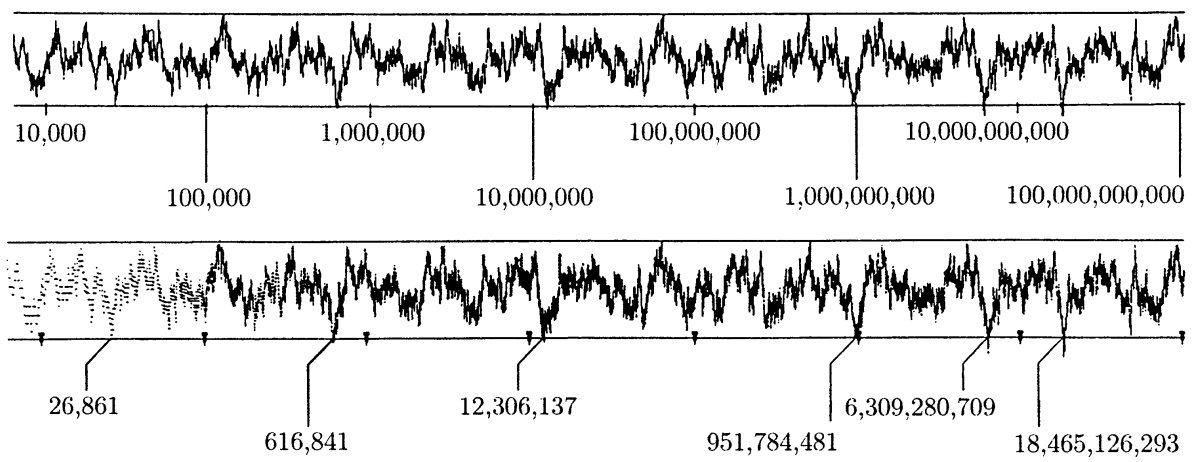

Figure 1. The upper graph was found in a few minutes using Theorem 1 with 12,000 zeroes of $L(s, \chi)$ for each point. The lower graph required an overnight run and produces exact results with the "Segmented Sieve of Eratosthenes" (see [4]). It gives the initial numerical values for each crossing region, and plots each point as closely as possible to its actual numerical value, which, of course, is beyond the resolution of the plot. Note the remarkable similarity of the two plots. For each power of ten interval, approximately 2,300 points were plotted. Note: The scale is logarithmic. Thus, the axis crossing at, for example, 616,841 in Figure 1 should occur, as it does, approximately $79 \%$ of the way from the mark at 100,000 to the mark at $1,000,000$, since the common logarithm of 616,841 is $5.79017 \cdots$.

\begin{tabular}{|l|l|}
\hline \multicolumn{2}{|c|}{ AXIS CROSSING REGION MINIMUM VALUES } \\
\hline As exactly determined [2] & $\begin{array}{l}\text { As found by applying Theorem 1 } \\
\text { using } 12,000 \text { zeroes of } L(s, \chi)\end{array}$ \\
\hline \hline 26861 & 26859 \\
623681 & 623705 \\
12366589 & $1.2366 \times 10^{7}$ \\
951867937 & $9.5199 \times 10^{8}$ \\
6345026833 & $6.3452 \times 10^{9}$ \\
18699356321 & $1.8696 \times 10^{10}$ \\
$1,488,478,427,089$ & $1.4898 \times 10^{12}$ \\
not known & $9.3190 \times 10^{12}$ \\
\hline
\end{tabular}

Figure 2. This table examines the minimum values at the first few axis crossing regions for $\pi_{4,3}(x)-\pi_{4,1}(x)$. The left column gives the exact values of these local minima as given in [2]. The "not known" entry refers to the axis crossing region that is in the vicinity of $9.3 \times 10^{12}$. This region was one of many additional ones that have been found using Theorem 1 . 


\section{REFERENCES}

[1] Carter Bays and Richard H. Hudson, On the fluctuations of Littlewood for primes of the form $4 n+1$, Math. Comp. vol. 32 (1978), pp. 281-286. MR 57:16174]

[2] Numerical and graphical description of all axis crossing regions for the moduli 4 and 8 which occur before $10^{12}$, Int. J. Math. \& Math. Sci., vol. 2(1979), pp. 111-119. MR 80h:10003

[3] - A new bound for the smallest $x$ with $\pi(x)>\operatorname{li}(x)$, to appear in Math. Comp.

[4] The segmented sieve of Erastosthenes and primes in arithmetic progressions to $10^{12}$, BIT, vol. 17, (1977), pp. 121-127. MR 56:5405

[5] H. Davenport, Multiplicative Number Theory (2nd ed.), Graduate Texts in Mathematics, vol. 74, Springer, Berlin, 1980. MR 82m:10001

[6] Richard H. Hudson, The mean behavior of primes in arithmetic progressions, J. Reine Angew. Math., vol. 296 (1977),pp. 80-99. MR 57:255

[7] _ Averaging effects on irregularities in the distribution of primes in arithmetic progressions, Math. Comp., vol. 44 (1985), pp. 561-571. MR 86h:11074

[8] J. Kaczorowski, Results on the distribution of primes, J. Reine Angew. Math. 446 (1994), 89-113. MR 95f: 11070

[9] - On the distribution of primes mod 4, Analysis 15 (1995), 159-171. MR 96h:11095

[10] John Leech, Note on the distribution of prime numbers, J. London Math. Soc., vol. 32 (1957), pp. 56-58. MR 18:642d

[11] Karl K. Norton, Upper bounds for $k$-th power coset representatives modulo n, Acta Arith., vol. 15 (1968/69) pp. 161-179. MR 39:1419

[12] Herman te Riele, On the sign change of the difference $\pi(x)-\operatorname{li}(x)$, Math. Comp., vol 48 (1986), pp. 667-681. MR 88a:11135

[13] Michael Rubinstein and Peter Sarnak, Chebyshev's Bias, Experimental Mathematics, vol. 3 (1994), pp. 173-197. MR 96d:11099

[14] Daniel Shanks, Quadratic residues and the distribution of primes, Math. Comp., vol 13 (1959), pp. 272-284. MR 21:7186

Department of Computer Science, University of South Carolina, Columbia, South Carolina 29208

E-mail address: bays@cs.sc.edu

Department of Mathematics, University of South Carolina, Columbia, South CarOLINA 29208

E-mail address: hudson@math.sc.edu 